TRANSACTIONS OF THE

AMERICAN MATHEMATICAL SOCIETY

Volume 354, Number 3, Pages 925-943

S 0002-9947(01)02854- 9

Article electronically published on October 24, 2001

\title{
SMALL PROFINITE STRUCTURES
}

\author{
LUDOMIR NEWELSKI
}

\begin{abstract}
We propose a model-theoretic framework for investigating profinite structures. We prove that in many cases small profinite structures interpret infinite groups. This corresponds to results of Hrushovski and Peterzil on interpreting groups in locally modular stable and o-minimal structures.
\end{abstract}

\section{INTRODUCTION}

Usually in mathematics by a profinite structure we mean the inverse limit of a system of finite structures of some kind. In this paper we propose a different (although equivalent) approach. This enables us to define in the context of profinite structures the counterparts of some basic notions of model theory and then to develop a "model theory" of so-called small profinite structures.

Given a profinite structure $U$ we adjoin to it some "imaginary" elements and form a structure $U^{e q}$. The relationship between $U$ and $U^{e q}$ is analogous to the relationship between a first-order structure $M$ and $M^{e q}$ in model theory. In $U^{e q}$ we define m-independence, which corresponds to forking independence in model theory, and m-normality, which describes the geometric character of m-independence similarly as 1-basedness describes the geometric character of forking. m-normality of a small profinite structure $U$ is related to local modularity of some geometries interpreted in $U$.

Usually the local modularity assumption implies existence of a definable group. In stable structures this was done by Hrushovski $[\mathrm{H}]$, in o-minimal structures by Peterzil [Pe, who considered the so-called CF-condition.

In this paper we prove an analogous result for small profinite structures. We prove that in the presence of a non-trivial locally modular geometry, in a small profinite structure $U$ there is a definable group related to this geometry.

The general scheme of the proof is the same as in $[\underline{\mathrm{H}}]$ and $[\mathrm{Pe}]$ : our definable group consists of certain definable functions. However the details are different. In [H] the construction uses stationary types (where forking geometry is similar to Zariski geometry). [Pe uses the fact that the order topology in a dense o-minimal structure is definably connected (and the intuitions from the real line).

In our set-up, a profinite structure carries a natural compact 0-dimensional (profinite) topology. So there is no counterpart of stationary types here and the topology is totally disconnected. Instead, to obtain a definable group we use the

Received by the editors August 30, 1999.

2000 Mathematics Subject Classification. Primary 03C45, 03C99; Secondary 51D20.

Key words and phrases. Profinite structure, m-independence, local modularity, combinatorial geometry.

Research supported by KBN grant 2 P03A 00216. 
smallness assumption. Actually, we reconstruct a definable group from an acltriangle in a small profinite m-normal structure. This corresponds to $\overline{\mathrm{BH}}$, where Bouscaren and Hrushovski reconstruct a group from an acl-triangle in a stable 1-based theory.

A natural example of a small profinite structure is any profinite structure interpretable in a small theory $T$ (the term interpretable used here is made precise in Section 2). By [Ne4, [Ne1, if $T$ is superstable with $<2^{\aleph_{0}}$ countable models, then any profinite structure $U$ interpretable in $T$ is m-normal, non-trivial and of finite $\mathcal{M}$-rank, so our results apply (in fact, in this case, the existence of a definable group in $U$ was proved earlier in [Ne3], by referring to the usual forking dependence in $T$, see also [Ne5]).

It is rather hard to construct a complicated small profinite structure. In [Ne2] some such constructions are shown (and these are groups), however the profinite groups occurring there are m-normal. I know no small profinite structure which is not m-normal. The results of this paper indicate that small profinite structures quite often contain definable groups.

\section{DEFINITIONS AND THE MAIN RESUlT}

We say that a topological space $U$ is profinite if there is a system $\mathcal{E}=\left\{E_{i}, i \in I_{U}\right\}$ of equivalence relations on $U$ such that

- each $E_{i}$ is closed (as a subset of $U^{2}$ ) and has finitely many classes;

- $\mathcal{E}$ is directed by the refinement relation, that is for all $i, j \in I_{U}$ there is some $t \in I_{U}$ such that $E_{t}$ refines both $E_{i}$ and $E_{j}$;

- the $E_{i}$-classes, $i \in I_{U}$, form a basis of the topology on $U$.

Then clearly $U$ is the inverse limit of the system $\bar{U}=\left\langle U / E_{i}, i \in I_{U}\right\rangle$ of finite discrete spaces. Also, the $E_{i}$-classes are clopen and $U$ is compact and 0 -dimensional.

Let $A u t_{0}^{*}(U)$ be the group of the homeomorphisms of $U$ induced by automorphisms of the system $\bar{U}$. In other words, $A u t_{0}^{*}(U)$ is the group of those homeomorphisms of $U$, that respect all the $E_{i}$ 's. The group $A u t_{0}^{*}(U)$ is the inverse limit of the system of groups $S\left(U / E_{i}\right), i \in I_{U}$, of permutations of $U / E_{i}$. So $A u t_{0}^{*}(U)$ is a profinite group acting continuously on $U$.

By a profinite structure we mean a profinite topological space $U$ described above, with a distinguished closed subgroup $A u t^{*}(U)$ of $A u t_{0}^{*}(U)$. From now on $U$ denotes a profinite structure in this sense, and we assume that the index set $I_{U}$ is countable (so $U$ is separable). Usually we suppress $A u t^{*}(U)$, speaking just of a profinite structure $U$.

$A, B$ denote usually finite subsets and $a, b$ finite tuples of elements of $U$ (or of $U^{e q}$, later). $A u t^{*}(U / A)=\left\{f \in A u t^{*}(U):\left.f\right|_{A}=i d\right\}$. So $A u t^{*}(U / A)$ is a closed subgroup of $A u t^{*}(U)$. We define the counterparts of the basic model-theoretic notions in $U$ referring only to the action of $A u t^{*}(U)$ on $U$, as if $U$ were a saturated model, or a monster model.

We say that $V \subseteq U^{n}$ is $A$-invariant (or, invariant over $A$ ) if $V$ is invariant under $A u t^{*}(U / A)$. "Invariant" means "invariant over $\emptyset "$ ". We say that $V \subseteq U^{n}$ is $A$-definable (or, definable over $A$ ) if $V$ is closed and $A$-invariant. "Definable" means "definable over some finite subset of $U$ ". So the family of sets definable in $U$ is not closed under complementation. Strictly speaking we should use the term "type-definable" instead of "definable" (see Example 2 in Section 2). 
Notice that equivalence relations $E_{i}, i \in I_{U}$, are 0-definable in $U$ (as subsets of $\left.U^{2}\right)$. Also we say that a function $f: U^{n} \rightarrow U^{m}$ is definable if its graph is definable.

Remark 1.1. Every definable function $f: U^{n} \rightarrow U^{m}$ is continuous.

We introduced definable sets in $U$ by means of the action of $A u t^{*}(U)$ on $U$. It turns out that vice versa, we can recover $A u t^{*}(U)$ from the family of 0 -definable sets in $U$.

Lemma 1.2. Assume $g: U \rightarrow U$ is a bijection. Then $g \in A u t^{*}(U) \Longleftrightarrow g$ preserves all 0 -definable subsets of $U^{n}, n<\omega$.

Proof $\Rightarrow$ is clear. $\Leftarrow:$ For any 0 -definable finite equivalence relation $E$ on $U$ let $\operatorname{Aut}(U / E)=\left\{\left.f\right|_{U / E}: f \in A u t^{*}(U)\right\}$. We know that $U$ is the inverse limit of the system $\left\langle U / E_{i}, i \in I_{U}\right\rangle$ of finite spaces. Let $S\left(U / E_{i}\right)$ denote the group of permutations of $U / E_{i}$. So $A u t\left(U / E_{i}\right)$ is a subgroup of $S\left(U / E_{i}\right)$ and $A u t_{0}^{*}(U)$ is the inverse limit of the system $S\left(U / E_{i}\right), i \in I_{U}$. Aut $*^{*}(U)$ is closed in $A u t_{0}^{*}(U)$, hence $A u t^{*}(U)$ is the inverse limit of the system $\operatorname{Aut}\left(U / E_{i}\right), i \in I_{U}$.

$g$ preserves each $E_{i}, i \in I_{U}$, so $g \in A u t_{0}^{*}(U)$. Hence we have that $g \in A u t^{*}(U)$ iff for every $i \in I_{U},\left.g\right|_{U / E_{i}} \in \operatorname{Aut}\left(U / E_{i}\right)$. Since $U / E_{i}$ is finite, there are finitely many invariant sets $S_{t} \subseteq\left(U / E_{i}\right)^{n_{t}}, t=1, \ldots, l$, such that for every $f \in S\left(U / E_{i}\right)$ we have $f \in \operatorname{Aut}\left(U / E_{i}\right)$ iff $f\left[S_{t}\right]=S_{t}$ for all $t$. But $\left.g\right|_{U / E_{i}}$ preserves all invariant sets in $U / E_{i}$. So we get $\left.g\right|_{U / E_{i}} \in \operatorname{Aut}\left(U / E_{i}\right)$ for any $i \in I_{U}$. Hence $g \in A u t^{*}(U)$.

Since we treat $U$ as a "monster model", model-theoretic types correspond to orbits here. So for $a \in U^{n}, o(a / A)$ denotes the orbit of $a$ under $A u t^{*}(U / A)$, called also the orbit of $a$ over $A$. $o(a)$ denotes $o(a / \emptyset)$ and is called the orbit of $a$. Since $A u t^{*}(U / A)$ acts continuously on $U, o(a / A)$ is closed and either finite or homeomorphic to the Cantor set (because $I_{U}$ is countable). $O_{n}(A)=\{o(a / A)$ : $\left.a \in U^{n}\right\} . O_{n}(A)$ is a compact 0-dimensional topological space (equipped with the hyperspace topology); it corresponds to the Stone space of types in model theory. $O_{n}(A)$ is homeomorphic to a closed subset of the Cantor set.

We say that $U$ is small if $O_{n}(\emptyset)$ is countable for every $n<\omega$. Equivalently, $O_{1}(A)$ is countable for every finite $A$. We are interested in describing definable sets in $U$. When $U$ is small then we can carry out in $U$ many standard model-theoretic arguments. From now on we usually assume that $U$ is a small profinite structure. (It is not clear if it is possible to extend the methods of this paper to the case of $U$, which is not small.)

Similarly as in model theory, we adjoin to $U$ certain "imaginary" objects. If $E$ is a 0 -definable equivalence relation on $U^{n}$, then $U / E$ denotes the set of $E$-classes, with the quotient topology. By smallness and [Ne6, Lemma 1.2], each such $E$ is an intersection of countably many 0-definable finite equivalence relations $E_{i}, i<\omega$. In particular, $U / E$ is a profinite topological space. For distinct relations $E$ we consider the sets $U / E$ disjoint.

Let $U^{e q}$ be the disjoint union of sets $U / E$, where $E$ is a 0 -definable equivalence relation on some $U^{n}$, equipped with the disjoint union topology. The sets $U / E$ are called the sorts of $U^{e q}$. We identify $U$ with $U /=$. (When $U$ is not small, we can still define $U^{e q}$ restricting ourselves to sorts $U / E$, where $E$ is a countable intersection of 0 -definable finite equivalence relations.)

$A u t^{*}(U)$ still acts continuously on $U^{e q}$. We extend the notions of definability, invariance, orbit to $U^{e q}$. For example, a definable set in $U^{e q}$ is a closed subset of 
a product of finitely many sorts of $U^{e q}$, invariant over some finite set. $F E$ denotes the set of all 0-definable finite equivalence relations in $U^{e q}$.

The transition from $U$ to $U^{e q}$ is similar to the transition from $M$ to $M^{e q}$ in model theory $\left[\mathrm{B}\right.$. In particular, $\left(U^{e q}\right)^{e q}$ essentially equals $U^{e q}$ and in $U^{e q}$ we have names for classes of all definable equivalence relations. Also, $U$ is small iff $U^{e q}$ is small. Similarly as in model theory, in $U^{e q}$ we have canonical names for definable sets.

This means that if $V$ is $A$-definable in $U^{e q}$, then there is some $V^{+} \in U^{e q}$ (called the name of $V$ ) such that for every $f \in A u t^{*}(U / A), f[V]=V$ iff $f\left(V^{+}\right)=V^{+}$. In particular, $V$ is definable over $V^{+}$. For example, assume $V$ is a 0 -definable set in $U$ and $V^{\prime}$ is a clopen subset of $V$. This implies that for some $E \in F E, V^{\prime}$ is a union of $E$-classes on $V$. Since $E$ has finitely many classes, we see that the orbit of the name $\left(V^{\prime}\right)^{+}$of $V^{\prime}$ is finite (that is, $\left(V^{\prime}\right)^{+} \in \operatorname{acl}(\emptyset)$ ).

Given a 0-definable equivalence relation $E$ in $U^{e q}$, we consider the $E$-classes in two ways. For a tuple $a$ of elements of suitable sorts of $U^{e q}, a / E$ denotes the $E$-class of $a$ as an element of $U^{e q}$ (or, if you like, a name of the $E$-class of $a$ ). $[a]_{E}$ denotes the $E$-class of $a$ as a subset of $U^{e q}$, that is $[a]_{E}=\left\{x \in U^{e q}: E(a, x)\right\}$. Clearly the set $[a]_{E}$ is definable over $a / E$ and $o(a /(a / E))=[a]_{E}$.

Assume $V$ is $A$-definable in $U^{e q}$. Then $V$ is a profinite topological space. $A u t^{*}(U / A)$ induces on $V$ a profinite structure, with the structure group $A u t^{*}(V)=$ $\left\{\left.f\right|_{V}: f \in \operatorname{Aut}^{*}(U / A)\right\}$.

Two profinite structures $U, V$ are called isomorphic, if there is a homeomorphism $f: U \rightarrow V$ (called an isomorphism) such that the pullback function $f^{*}$ maps $A u t^{*}(V)$ onto $A u t^{*}(U)$, then $f^{*}$ is an isomorphism of the structure groups.

Lemma 1.3. Assume $U, V$ are profinite structures and $f: U \rightarrow V$ is a bijection. Then the following conditions are equivalent.

(1) $f$ is an isomorphism.

(2) For every $X \subseteq U^{n}, X$ is 0-definable in $U$ iff $f[X]$ is 0-definable in $V$.

Proof. (1) $\rightarrow(2)$. We know that $f$ is a homeomorphism. We must prove that

$X$ is invariant in $U \Longleftrightarrow f[X]$ is invariant in $V$.

It is enough to prove $\Rightarrow$. Assume $g \in A u t^{*}(V)$. By (1), $f^{*}(g)=f^{-1} g f \in$ $A u t^{*}(U)$, so $f^{-1} g f[X]=X$. This gives $g[f[X]]=f[X]$, as needed.

$(2) \rightarrow(1)$. First we show that $f$ is a homeomorphism. The topology on $V$ is induced by a system $\mathcal{E}_{V}=\left\{E_{i}, i \in I_{V}\right\}$ of finite closed equivalence relations. Each $E_{i}$ is 0-definable, so the equivalence relations $E_{i}^{\prime}=f^{-1}\left[E_{i}\right]$ are also 0-definable, hence have clopen equivalence classes. This shows that $f$ is continuous. Similarly we show that $f^{-1}$ is continuous.

Now let $g \in A u t^{*}(V)$. We must show that $f^{*}(g)=f^{-1} g f \in A u t^{*}(U)$. We use the criterion from Lemma 1.2. Let $X \subseteq U^{n}$ be 0 -definable. It is enough to show that $f^{*}(g)[X]$ is 0 -definable. This follows easily from the assumptions and Lemma 1.2 .

We say that a profinite structure $V$ is interpretable in $U$ if there is a continuous 1-1 mapping $f$ of $V$ onto a set $f[V]$ definable in $U^{e q}$ over some finite set $A$, such that all 0 -definable sets in $V$ are mapped to $A$-definable sets in $f[V]$. Equivalently, the pullback function maps $A u t^{*}(U / A)$ onto a closed subgroup of $A u t^{*}(V)$.

Similarly as in model theory we define the algebraic and definitional closure in $U^{e q}$. For finite $A \subseteq U^{e q}, \operatorname{acl}(A)$ is the union of all finite orbits in $U^{e q}$, over $A$. 
$\operatorname{dcl}(A)$ is the union of all 1-element orbits in $U^{e q}$, over $A$. When $A$ is infinite, $\operatorname{acl}(A)=\bigcup\left\{\operatorname{acl}\left(A^{\prime}\right): A^{\prime} \subseteq A\right.$ is finite $\}$ and $\operatorname{dcl}(A)$ is defined similarly in this case. For example, $U^{e q} \subseteq d c l(U)$. $U$ may be regarded as a type-definable set in some first-order structure $N$, consisting of some infinite tuples of elements of $a c l(\emptyset)$ in $N$ (see Examples 2 and 3 from Section 2). Then, in the sense of forking in $N, U^{e q}$ is independent. This implies that $a c l$ and $d c l$ are closely related in $U^{e q}$.

Remark 1.4. $\operatorname{acl}(A)=d \operatorname{cl}(A \cup \operatorname{acl}(\emptyset))$.

Proof. It is enough to prove $\subseteq$. Let $a \in \operatorname{acl}(A)$. So $o(a / A)$ is finite. Choose $E \in E F$ with $[a]_{E} \cap o(a / A)=\{a\}$. So $a / E \in \operatorname{acl}(\emptyset)$ and $a \in \operatorname{dcl}(A \cup\{a / E\})$.

When $A, B \subseteq U^{e q}$ are finite, then $o(a / A \cup B)$ is a closed subset of $o(a / A)$ and is either open in $o(a / A)$ or nowhere dense. In the first case we say that

$$
a \text { is } m \text {-independent from } B \text { over } A\left(a^{m} B(A)\right) \text {. }
$$

We apply this definition also when $A, B$ are finite tuples of elements of $U^{e q}$.

Lemma 1.5. (1) m-independence is symmetric and transitive.

(2) $a \in \operatorname{acl}(A)$ implies $a^{m} \mathbb{I} B(A)$ for every finite $B$.

(3) For every finite $a, A, B \subseteq U^{e q}$, there is some $a^{\prime} \in o(a / A)$ with $a^{\prime^{m}} \downarrow B(A)$.

Proof. The proof is the same as in [Ne2, $\mathbb{N e 4}$. In (3) we use the fact, that by smallness, for any orbit $X$ over $A$, the union of all orbits over $A \cup B$ open in $X$ is dense in $X$.

To measure orbits we assign to each orbit its $\mathcal{M}$-rank, which is an ordinal or $\infty$. More precisely, $\mathcal{M}$ is the minimal function defined on all orbits in $U^{e q}$ over finite sets, with values in $\operatorname{Ord} \cup\{\infty\}$, such that for all finite $a, A \subseteq U^{e q}$ we have

$$
\begin{aligned}
& \mathcal{M}(o(a / A)) \geq \alpha+1 \Longleftrightarrow \mathcal{M}(o(a / B)) \geq \alpha \text { for some finite } B \supseteq A \text { with } \\
& o(a / B) \text { nowhere dense in } o(a / A) .
\end{aligned}
$$

$\mathcal{M}(a / A)$ abbreviates $\mathcal{M}(o(a / A))$ and $\mathcal{M}(a)$ is $\mathcal{M}(a / \emptyset)$.

We could define $\mathcal{M}$-rank inside $U$ (without referring to $U^{e q}$ ). It turns out however that for $a, A \subseteq U, \mathcal{M}(a / A)$ does not depend on whether in the definition of $\mathcal{M}$-rank we consider all finite sets $B \subseteq U^{e q}$ or restrict ourselves to subsets of $U$ [Ne2]. We say that $U$ is $\mathrm{m}$-stable if $\mathcal{M}(a / A)<\infty$ for all finite $a, A \subseteq U$ (equivalently, for all finite $a, A \subseteq U^{e q}$.

$\mathcal{M}$-rank satisfies Lascar inequalities

$$
\mathcal{M}(a / A b)+\mathcal{M}(b / A) \leq \mathcal{M}(a b / A) \leq \mathcal{M}(a / A b) \oplus \mathcal{M}(b / A) .
$$

In stable model theory we discern a geometric property of forking independence called 1-basedness. This property is equivalent (in the finite rank case) to local modularity of forking geometry on any $U$-rank 1 type. In o-minimal structures the corresponding property is called the CF-condition $[\mathrm{Pe}$, it corresponds to local modularity of the acl-geometry on an o-minimal structure.

The corresponding property of m-independence in small profinite structure $U$ is called m-normality. We say that $U$ is m-normal if for every finite $a, b \subseteq U^{e q}$ there is some $c \in \operatorname{acl}(a) \cap a c l(b)$ with $a^{m} \amalg b(c)$. In [Ne4] I was considering this property in the context of a small theory. However, the same proofs work in small profinite structures. So by $\mathrm{Ne} 2 \mathrm{Ne} 4$, the following conditions are equivalent to m-normality of $U$ : 
(1) For every finite $a, A \subseteq U$, for some clopen neighborhood $V$ of $a$, there are finitely many conjugates of $V \cap o(a / A)$ under $A u t^{*}(U / a)$.

(2) The same as (1), but for finite $a, A \subseteq U^{e q}$.

If $U$ is m-normal and $\mathrm{m}$-stable, then $\mathcal{M}$-rank is finite in $U$ [Ne4].

To explain the geometric meaning of m-normality we interpret some geometries in $U$. Assume $X$ is an orbit in $U^{e q}$ (over $\emptyset$ ), with $\mathcal{M}(X)=1$. Then m-dependence on $X$ equals acl-dependence and is a pregeometry. Assume $D$ is a countable dense subset of $X$. We say that $X$ is locally modular, if the acl $_{D}$-geometry on $X$ is modular (the choice of $D$ is irrelevant). Here $\operatorname{acl}_{D}(B)=\operatorname{acl}(D \cup B), \operatorname{acl}_{D}$ is the algebraic closure localized at $D$. The case of an orbit $X$ over a finite set $A$ is handled similarly. Unfortunately, unlike in the case of a stationary type, the acl-geometry on $X$ is not homogeneous, however localizing this geometry at a dense subset makes it homogeneous. This is the reason why we need the set $D$. For more details see [Ne3, Section 3].

It is easy to see [Ne4] that in an m-normal small profinite structure, every orbit of $\mathcal{M}$-rank 1 is locally modular. It turns out, that the connection between m-normality and local modularity is even closer. To explain this we define some notions.

Assume $X$ is an orbit (over $A$ ). We say that $a \in X$ is generic over $B$ iff $a^{m} \downarrow B(A)$ (generic elements exist by Lemma 1.5(3)). As in forking theory, we say that two orbits : $X$ (over $A$ ) and $Y$ (over $B$ ) are m-orthogonal, if for every finite $C \supseteq A \cup B$, for all $a \in X$ and $b \in Y$ generic over $C$, we have $a^{m} \mathcal{\perp} b(C)$. If $X$ and $Y$ are over the same set $A$, we say that $X, Y$ are almost m-orthogonal if we have $\left.a^{m}\right\rfloor b(A)$ for all $a \in X$ and $b \in Y$.

We say that $U$ has weak coordinatization, if

$(*)$ for every infinite orbit $X$ in $U^{e q}$ (over $A$ ), there is some finite $B \supseteq A$ and $a \in X$ generic over $B$, such that for some $c \in \operatorname{acl}(B a)$ we have $\mathcal{M}(c / B)=1$

(in other words, $X$ is m-nonorthogonal to an orbit of $\mathcal{M}$-rank 1). We say that $U$ has full coordinatization, if $(*)$ holds for $B=A$.

Theorem 1.6 (essentially [Ne4]). Assume $U$ is a small profinite structure of finite $\mathcal{M}$-rank. Then the following conditions are equivalent.

(1) $U$ is m-normal.

(2) $U$ has weak coordinatization and every $\mathcal{M}$-rank 1 orbit in $U^{e q}$ is locally modular.

(3) $U$ has full coordinatization and every $\mathcal{M}$-rank 1 orbit in $U^{\text {eq }}$ is locally modular.

We say that an orbit of $\mathcal{M}$-rank 1 is non-trivial, if the associated pregeometry is non-trivial (that is, after the localization, a "line" has at least 3 points, here notice again that the localized geometry is homogeneous). m-normal small profinite structures may be thought of as "geometrically simple" (just like 1-basedness is a kind of geometric simplicity of forking geometry). Surprisingly, no small profinite structure is known, which is not m-normal.

We say that $G$ is an $A$-definable group in $U^{e q}$ if $G$ and the group operations of $G$ are $A$-definable in $U^{e q}$. We say that $a \in G$ is m-generic over $A$ if $o(a / A)$ is open in $G$. By smallness, m-generic elements (over $A$ ) are dense in $G$. We define $\mathcal{M}(G)$ (the $\mathcal{M}$-rank of $G$ ) as $\mathcal{M}(a / A)$ for any $a \in G$ m-generic over $A$ (this does 
not depend on the choice of $a$ ). Every group $G$ definable in $U^{e q}$ is profinite and locally finite Ne6.

In the case of a stable structure or an o-minimal structure, Hrushovski $[\mathrm{H}]$ and Peterzil $[\mathrm{Pe}$ respectively proved that local modularity implies existence of a definable group in a given structure. In this paper we prove a counterpart of these results for small profinite structures. The main result is the following theorem.

Theorem 1.7. If $U$ is a small profinite structure and $\mathcal{O}$ is a non-trivial locally modular orbit in $U$ (over $\emptyset$ ), of $\mathcal{M}$-rank 1 , then some open subset $\mathcal{O}^{\prime}$ of $\mathcal{O}$ is a definable group.

\section{EXAMPLES}

In this section we show three examples of profinite structures. These examples illustrate and justify the definitions from Section 1.

Example 1. Assume $G$ is a profinite group, that is the inverse limit of a system $\bar{G}=\left\langle G_{i}, f_{j i}\right\rangle_{j>i \in I}$ of finite groups, indexed by a (countable) directed set $I$. We have natural projections $f_{\infty i}: G \rightarrow G_{i}$, that we may assume are surjective.

For $i \in I$ let $E_{i}$ be the equivalence relation on $G$ induced by $f_{\infty i}$, that is, $E_{i}(x, y)$ holds iff $f_{\infty i}(x)=f_{\infty i}(y)$. We see that the equivalence relations $E_{i}, i \in I$, generate the profinite topology on $G$. Let $A u t_{0}^{*}(G)$ be the set of homeomorphisms of $G$ that preserve all the $E_{i}$ 's, and let $A u t^{*}(G)$ be the group of automorphisms of $G$ that are induced by automorphisms of the system $\bar{G}$. We see that $A u t^{*}(G)$ is a closed subgroup of $A u t_{0}^{*}(G)$.

So $G$ with structure group $A u t^{*}(G)$ becomes a profinite structure in the sense of Section 1. Notice that the group operations are 0-definable in $G$, so $G$ is a profinite group in the sense of Section 1. According to the definition from Section 1, $G$ is small (as a profinite structure) iff on every $G^{n}$ there are countably many orbits of $A u t^{*}(G)$. For example, the product of countably many copies of $Z_{2}$ is a small profinite group. A major open question about small profinite groups is whether any such group contains an open abelian subgroup ([Ne6] contains some related results). The expected answer is "no", however it is hard to find a counterexample.

Example 2. Assume $M$ is a multifinite structure, that is a many-sorted structure in some language $L$, with all the sorts finite. Notice that $M$ is $\kappa$-saturated for every $\kappa$. In fact, we may regard $M$ as a monster model of its theory. We equip $M$ with the discrete topology. Let $I$ be a countable index set and $x_{I}=\left\langle x_{i}, i \in I\right\rangle$ be a tuple of variables of specified sorts $M_{i}, i \in I$. Let $\Phi\left(x_{I}\right)$ be a type in variables $x_{I}$, without parameters. Let $U \subseteq \prod_{i \in I} M_{i}$ be the set of realizations of $\Phi$ in $M$. So $U$ is a closed subset of $\prod_{i \in I} M_{i}$ (with the product topology). In fact, $U$ is a profinite topological space.

Notice that any profinite structure $V$ may be regarded as a type-definable set in some multi-finite structure $N$ in the above way, so that the 0 -definable subsets of $V^{n}$ (in the sense of a profinite structure) are precisely the 0-type-definable subsets of $V^{n}$ (in the sense of $N$ ).

Indeed, let $\mathcal{E}=\left\{E_{i}, i \in I_{V}\right\}$ be a system of 0-definable equivalence relations generating the topology on $V$. Let $V_{i}=V / E_{i}$ and let $A u t\left(V_{i}\right)$ be the restriction of $A u t^{*}(V)$ to $V_{i}$. $I_{V}$ is directed by the relation $i \leq j$ iff $E_{j}$ refines $E_{i}$. For $j \geq i$ let $f_{j i}: V_{j} \rightarrow V_{i}$ be the function mapping $x / E_{j}$ to $x / E_{i}$. 
On each set $V_{i}$ we define an $L_{i}$-structure (for some finite language $L_{i}$ ) so that $\operatorname{Aut}\left(V_{i}\right)$ is the group of automorphisms of $V_{i}$ as the $L_{i}$-structure (as in the proof of Lemma 1.2). Let $L$ be a many-sorted language with the set of sorts $I_{V}$, containing the symbols of $L_{i}, i \in I_{V}$, as symbols of sort $i$, and function symbols $f_{j i}$ as symbols of functions between sort $j$ and $i$.

We define a multifinite $L$-structure $N$ as follows. The sort $i$ of $N$ is the set $V_{i}$, togehter with its $L_{i}$-structure. Moreover, in $N$ there are functions $f_{j i}$ between sets $V_{j}$ and $V_{i}, j \geq i \in I_{V} . V$ may be identified with the set of $I_{V}$-tuples from $\prod_{i \in I_{V}} V_{i}$ satisfying the type $\left\{f_{j i}\left(x_{j}\right)=x_{i}: j \geq i \in I_{V}\right\}$.

Clearly, $\operatorname{Aut}^{*}(V)=\left\{\left.f\right|_{V}: f \in \operatorname{Aut}(N)\right\}$. Since $N$ is a monster model, we see that the 0-definable subsets of $V^{n}$ (in the sense of profinite structure $V$ ) are precisely the 0-type-definable subsets of $V^{n}$ (in the sense of $N$ ).

Now consider the type-definable set $U$ from the beginning of Example 2. We see that any profinite structure may be considered in this way. Now we show however, that the approach from Section 1 is equivalent to the approach in Example 2. Suppose we are interested in subsets of $U^{n}$ type-definable over a finite set of parameters from $M \cup U$. The next lemma shows we may disregard parameters from $M$. This lemma corresponds to Shelah's definability lemma in stable model theory.

Lemma 2.1. Assume $V \subseteq U^{n}$ is type-definable over a finite set $A \subseteq M \cup U$. Then $V$ is type-definable over a finite set $B \subseteq U$.

Proof. $A=A_{0} \cup A_{1}$, where $A_{0}$ is contained in finitely many sorts $M_{1}, \ldots, M_{n}$ of $M$ and $A_{1} \subseteq U$. The sorts of $M$ are finite, so there are finitely many conjugates $A_{0}^{1}, \ldots, A_{0}^{t}$ of $A_{0}$ over $A_{1}$. For each $A_{0}^{i}$ let $V^{i}$ be the corresponding conjugate of $V$. Choose a finite set $B \subseteq V^{1} \cup \cdots \cup V^{t}$ such that for $1 \leq i \neq j \leq t, B \cap V^{i} \neq B \cap V^{j}$. We see that $V$ is invariant under $\operatorname{Aut}\left(M / A_{1} \cup B\right)$. $V$ is also closed, hence by saturation of $M, V$ is type-definable over $A_{1} \cup B$.

By saturation of $M$, a set $V \subseteq U^{n}$ is type-definable over $A$ iff $V$ is closed and invariant under $\operatorname{Aut}(M / A)$. By Lemma 2.1 we see that we can recover all the definable subsets of $U^{n}$ using the topology of $U$ and the action of $A u t^{*}(U)=\left\{\left.f\right|_{U}\right.$ : $f \in A u t(M)\}$ on $M$. So the type-definable subsets of $U^{n}$ (in the sense of $M$ ) are precisely the definable subsets of $U$ (in the sense of the profinite structure $U$ with structure group $\left.A u t^{*}(U)\right)$.

The approach from Section 1 seems better to me: we do not have to bother about the underlying language or multifinite structure.

Example 3. This example is the real motivation to consider small profinite structures. Assume $T=T^{e q}$ is a countable complete first-order theory and $M$ is an $\aleph_{1}$-saturated $\aleph_{1}$-strongly homogeneous model of $T$. Assume $X$ is a 0 -type-definable subset of $M$ and $f_{i}, i \in I$, are countably many definable functions, $f_{i}: X \rightarrow \operatorname{acl}(\emptyset)$. An $I$-tuple of the form $\left\langle f_{i}(a): i \in I\right\rangle$, where $a \in X$, was called in Ne2 a $*$-algebraic $*$-finite tuple. Such tuples play the role of imaginaries for $\mathrm{m}$-independence in a small stable theory [Ne5].

Let $U=\left\{\left\langle f_{i}(a): i \in I\right\rangle: a \in X\right\} \subseteq[\operatorname{acl}(\emptyset)]^{I}$. So $U$ is a type-definable set of $I$-tuples in $M$. We equip $\operatorname{acl}(\emptyset)$ with the discrete topology. Then $U$ is a closed subset of $[\operatorname{acl}(\emptyset)]^{I}$ (with the product topology). We are interested in subsets of $U$ type-definable over finite sets of parameters from $U$. Then type-definable sets in $U$ (in the sense of $M$ ) correspond precisely to definable sets in profinite structure $U$ with the structure group $A u t^{*}(U)=\left\{\left.f\right|_{U}: f \in A u t(M)\right\}$. 
We say that the profinite structure $U$ described above is interpretable in $T$. Also, if $T$ is small, then any profinite structure interpretable in $T$ is small.

Assume $T$ is superstable with $<2^{\aleph_{0}}$ countable models. I defined m-independence and $\mathcal{M}$-rank in models of $T$ and proved that $T$ is (1) m-stable, (2) of finite $\mathcal{M}$ rank and (3) m-normal [Ne5. If $T$ satisfies (1), (2) or (3), then any profinite structure interpretable in $T$ also has the same property. This is the reason why these notions were so defined in Section 1, for small profinite structures. Also, many arguments regarding m-independence in models of $T$ transfer directly to small profinite structures (so we were referring to $[\mathrm{Ne} 2, \mathrm{Ne} 4$ in Section 1).

Many open questions about m-independence in small (stable) theories translate directly into questions about small profinite structures. We state some of them.

Conjecture 2.2 (related to the $\mathcal{M}$-gap conjecture [Ne2]). There is no orbit $X$ in a small profinite structure with $\omega \leq \mathcal{M}(X)<\infty$.

Every small theory known is m-normal, although presumably non m-normal small theories exist. This is equivalent to existence of a small profinite structure which is not m-normal (and this is an open problem). The $\mathcal{M}$-gap conjecture is true for small m-normal theories [Ne4] (hence also for small m-normal profinite structures).

One of the consequences of m-normality for a small profinite group $G$ is that $G$ is abelian-by-finite. So any example of a small profinite group $G$ without an open abelian subgroup would be non m-normal. That is why we state this problem about small profinite groups.

Similarly, the existence of a small theory, for which the notions of independence induced by Baire category ( $\mathrm{m}$-independence) and Lebesgue measure $(\mu$ independence) differ, is equivalent to the existence of small profinite structure with this property (for details on the comparison between m-independence and $\mu$-independence see [Ne2]).

This justifies our interest in small profinite structures. However I also believe that small profinite structures may become an independent object of study, since no knowledge of model theory is needed to investigate them.

\section{The PROOF}

In this section we prove the main result of this paper which is the interpretation of a group in some small profinite structures (Theorem 1.7). We will use the properties of small profinite structures from Section 1. The arguments involving $\mathrm{m}$-independence are similar to those about forking independence.

Assume $\mathcal{O}$ is an $\mathcal{M}$-rank 1 locally modular non-trivial orbit, definable over $\emptyset$ in a small profinite structure $U$. Let $U^{\prime}$ be the small profinite structure induced on the profinite space $\mathcal{O}$ by $U$ (that is, $A u t^{*}\left(U^{\prime}\right)$ is the restriction of $A u t^{*}(U)$ to $\mathcal{O}$ ).

By the Lascar inequalities, $U^{\prime}$ is a small profinite structure of finite $\mathcal{M}$-rank. Every $\mathcal{M}$-rank 1 orbit in $\left(U^{\prime}\right)^{e q}$ is m-nonorthogonal to $\mathcal{O}$, hence is also locally modular. More generally, every infinite orbit in $\left(U^{\prime}\right)^{e q}$ is m-nonorthogonal to $\mathcal{O}$, that is $U^{\prime}$ has weak coordinatization. By Theorem 1.6 this implies that $U^{\prime}$ is mnormal.

So without loss of generality, replacing $U$ by $U^{\prime}$, we may assume that $U$ is a small profinite structure, which is m-normal (and even has finite $\mathcal{M}$-rank). We work in $U^{e q}$. We will be working with sets definable in $U^{e q}$ over finitely many parameters. In model theory we often name a given set of parameters (expanding the language 
by some new constants), so as to consider only 0-definable sets or types over $\emptyset$. Here we have a similar procedure. Assume $A$ is a finite subset of $U^{e q}$. Then $A u t^{*}(U / A)$ is a closed subgroup of $A u t^{*}(U)$. $U$ with the structure group $A u t^{*}(U / A)$ is again a small profinite m-normal structure of finite $\mathcal{M}$-rank, however every $A$-definable set in the structure $\left(U, A u t^{*}(U)\right)$ becomes 0-definable in $\left(U, A u t^{*}(U / A)\right)$. We call the transition from $\left(U, A u t^{*}(U)\right)$ to $\left(U, A u t^{*}(U / A)\right)$ "naming parameters" from $A$.

In the proof of Theorem 1.7 we use the following lemma.

Lemma 3.1. Assume $U$ is a small profinite structure and $X$ is an orbit in $U$, over $\emptyset$. Then $\operatorname{acl}(a) \cap X$ is finite for every $a \in X$.

Proof. For $a \in X$ let $X_{a}=\operatorname{acl}(a) \cap X$. We see that the sets $X_{a}, a \in X$, are conjugate. Fix an $a \in X$.

Let $Y_{a}$ be the topological closure of $X_{a}$. By smallness, $Y_{a}$ and $X_{a}$ are countable unions of orbits over $a$; the orbits contained in $X_{a}$ are finite, hence $X_{a}$ is countable. The union of the orbits over $a$ that are open in $Y_{a}$ is dense in $Y_{a}$. Such orbits meet $X_{a}$, hence are contained in $X_{a}$, and are finite. It follows that

(a) the set of isolated points of $Y_{a}$ is dense in $Y_{a}$.

Next we prove that for $b \in Y_{a}$ we have

(b) $Y_{b}=Y_{a}$.

First we show that $X_{b} \subseteq Y_{a}$ (which implies that $Y_{b} \subseteq Y_{a}$ ). Indeed, if $c \in X_{b}$, then $o(b c)$ is a closed subset of $X \times X$ projecting onto both axes, with vertical sections finite. If we take $a^{\prime} \in X_{a}$ sufficiently close to $b$, then for some $c^{\prime}$ close to $c$ we have $a^{\prime} c^{\prime} \in o(b c)$, hence $c^{\prime} \in \operatorname{acl}\left(a^{\prime}\right)$, which yields $c^{\prime} \in X_{a}$ (because acl is a closure operator). So $c \in \operatorname{cl}\left(X_{a}\right)=Y_{a}$.

Now let $E_{i}, i \in I$, be a countable system of 0 -definable finite equivalence relations generating the topology on $U$. Since $Y_{a}$ and $Y_{b}$ are conjugate, for every $i, Y_{a}$ and $Y_{b}$ meet the same number of $E_{i}$-classes. This implies that $Y_{b}=Y_{a}$.

Consider an isolated point $b \in Y_{a}$. By (b), $Y_{b}=Y_{a}$. Since $Y_{b}$ and $Y_{a}$ are conjugate, we get that $a$ is isolated in $Y_{a}$. Similarly it follows that any $c \in Y_{a}$ is isolated in $Y_{a}$, so $Y_{a}$ and $X_{a}$ are finite.

From now on we fix a non-trivial locally modular orbit $\mathcal{O}$ in $U$ (over $\emptyset$ ), with $\mathcal{M}(\mathcal{O})=1$. Also, we assume $U$ is m-normal. We are going to find a definable group in $U$, which is an open subset of $\mathcal{O}$.

First, we will define a group structure on an open subset of $\mathcal{O}$ over some unspecified finite number of parameters. Later (in Section 4) we restrict this number to 1 parameter from $\mathcal{O}$ and some finite number of parameters algebraic over $\emptyset$.

We say that $A=\{a, b, c\}$ is a $d c l$-triangle, if for every $x \in A$ we have $x \in$ $\operatorname{dcl}(A \backslash\{x\}) \backslash \operatorname{acl}(\emptyset)$ and every two elements of $A$ are m-independent. Replacing $d c l$ by $a c l$ in the last sentence we get the definition of an acl-triangle.

Lemma 3.2. After naming finitely many parameters, in $\mathcal{O}$ there is an acl-triangle.

Proof. Since $\mathcal{O}$ is non-trivial, there are acl-dependent $a_{0}, \ldots, a_{n} \in \mathcal{O}$ (for some $n \geq 2$ ) such that every proper subset of $\left\{a_{0}, \ldots, a_{n}\right\}$ is acl-independent. Name $a_{3}, \ldots, a_{n}$. Then $\left\{a_{0}, a_{1}, a_{2}\right\}$ becomes an acl-triangle.

We name the finitely many parameters from Lemma 3.2. Let $\{a, b, c\}$ be an acl-triangle in $\mathcal{O}$. Theorem 1.7 follows from the following more general result on recovering a group from an acl-triangle in a small profinite m-normal structure $U$. 
This result corresponds to $[\mathrm{BH}]$, where a group is constructed from an acl-triangle in a stable 1-based theory.

Theorem 3.3. Assume $\{a, b, c\}$ is an acl-triangle in a small profinite m-normal structure $U$. Then there is a group $G$ in $U$, which is open in o(a), a is the neutral element of $G$ and $G$ is definable over a and acl $(\emptyset)$.

Proof. In the proof we may freely name finitely many elements of $\operatorname{acl}(\emptyset)$. So by Remark 1.4 we may assume that $\{a, b, c\}$ is a $d c l$-triangle. By Lemma 3.1, naming some elements of $\operatorname{acl}(\emptyset)$, we may assume that for every $x \in\{a, b, c\}$ we have $a c l(x) \cap$ $o(x)=\{x\}$.

Let $b^{+}$be the name of $o(b / a)$. Since $o(b / a)$ is clopen in $\mathcal{O}$ (hence for some $E \in$ $F E, o(b / a)$ is a finite union of $E$-classes on $o(b))$, we have that $b^{+} \in \operatorname{acl}(\emptyset) \cap d c l(a)$. We see that

$$
o\left(b / b^{+}\right)=o\left(b / a b^{+}\right)=o(b / a) .
$$

So after naming $b^{+}$we have $o(b / a)=o(b)$, which gives $o(a b)=o(a) \times o(b)$.

Let $X=o(a), Y=o(b), Z=o(c)$ and $\Gamma=o(a b c) \subseteq X \times Y \times Z$. For every $x \in X$, the section $\Gamma_{x} \subseteq Y \times Z$ of $\Gamma$ at $x$ is the graph of an $x$-definable function $f_{x}: Y \rightarrow Z$. Throughout, we identify definable functions with the names of their graphs, and so we treat such functions as elements of $U^{e q} . f_{x}$ is 1-1 (because $\{a, b, c\}$ is a $d c l$-triangle). We have that $f_{x}$ and $x$ are interdefinable.

Indeed, we have $f_{x} \in d c l(x)$ by definition. To prove that $x \in d c l\left(f_{x}\right)$, we may assume without loss of generality that $x=a$. Suppose $x^{\prime} \in o\left(a / f_{a}\right)$, we will prove $x^{\prime}=a$. We have $f_{x^{\prime}}=f_{a}$ and $o(a b)=o\left(x^{\prime} b\right)$. There is precisely one $c^{\prime}$ with $o(a b c)=o\left(x^{\prime} b c^{\prime}\right)$, and $c^{\prime}=f_{x^{\prime}}(b)=f_{a}(b)=c$. So $o(a b c)=o\left(x^{\prime} b c\right)=\Gamma$. As $\{a, b, c\}$ is a $d c l$-triangle, we get $x^{\prime}=a$.

The set $f_{a}[Y]$ is a clopen subset of $Z$ (more precisely, $f_{a}[Y]=o(c / a)$ ). Let $X_{0}$ be the orbit of $a$ over the name of $f_{a}[Y], X_{0}$ is a clopen subset of $X$. For $x, x^{\prime} \in X_{0}$, the functions $f_{x}$ and $f_{x^{\prime}}$ are bijections between $Y$ and $f_{a}[Y]$, so $g_{x^{\prime} x}:=f_{x^{\prime}}^{-1} f_{x}$ is a bijection mapping $Y$ onto $Y$.

At this point, in the case of a 1-based stable theory, essentially the germs of functions $g_{x^{\prime} x}$ generate a type-definable group. Here we do not have germs at hand, instead we will construct a group from carefully chosen pieces $h_{x^{\prime} x}$ of $g_{x^{\prime} x}$.

Fix $a^{\prime} \in X_{0}$ generic over $a, b$. So $a^{m} a^{\prime}$ and $b^{m} g_{a^{\prime} a}$. Let $b^{\prime}=g_{a^{\prime} a}(b)=f_{a^{\prime}}^{-1}(c)$. Let $V=o\left(b b^{\prime} / g_{a^{\prime} a}\right)$.

So $V$ is a (relatively) clopen subset of the graph of $g_{a^{\prime} a}$. By m-normality, there is some clopen neighbourhood $V^{\prime} \subseteq V$ of $b b^{\prime}$ such that

(*) the name of $V^{\prime}$ is algebraic over $b b^{\prime}$.

If we take a smaller $V^{\prime}$, then $(*)$ remains true. So we may assume $V^{\prime}$ is the graph of the function $\left.g_{a^{\prime} a}\right|_{[b]_{E} \cap Y}$ for some $E \in F E$. Let $Y_{0}=[b]_{E} \cap Y$ and $h_{a^{\prime} a}=\left.g_{a^{\prime} a}\right|_{Y_{0}}$. We name $Y_{0}^{+}$(the name of $\left.Y_{0}\right)$.

Claim 3.4. (1) Except for $\{a, b, c\}$ and $\left\{a^{\prime}, b^{\prime}, c\right\}$, every 3-element subset of $\{a, b, c$, $\left.a^{\prime}, b^{\prime}\right\}$ is m-independent.

(2) $\left\{b, b^{\prime}, h_{a^{\prime} a}\right\}$ is an acl-triangle.

(3) $\left\{a, a^{\prime}, h_{a^{\prime} a}\right\}$ is an acl-triangle.

Proof. (1) By assumption, $\{a, b, c\}$ and $\left\{a^{\prime}, b^{\prime}, c\right\}$ are $d c l$-triangles and $\left\{a, a^{\prime}, b\right\}$ is m-independent. For example, we prove that $\left\{a^{\prime}, b, c\right\}$ is m-independent (the other cases are similar). 
Since $\{a, b, c\}$ is a $d c l$-triangle, there is a $b$-definable bijection $j: o(c / b) \rightarrow o(a / b)$ and moreover $o(c / b)$ and $o(a / b)$ are open in $o(c), o(a)$ respectively. So if $\left\{a^{\prime}, b, c\right\}$ is not m-independent, then $c^{m} \mathbb{L} a^{\prime} b$, hence $o\left(c / a^{\prime} b\right)$ is nowhere dense in $o(c / b)$. Hence $o\left(a / a^{\prime} b\right)=j\left(o\left(c / a^{\prime} b\right)\right)$ is nowhere dense in $o(a / b)$, contradicting $\left.a^{m}\right\rfloor a^{\prime}(b)$.

(2) It is enough to show that every proper subset of $\left\{b, b^{\prime}, h_{a^{\prime} a}\right\}$ is m-independent. $b^{m} \underline{1}^{\prime}$, because (by (1)) $\left\{a, b, b^{\prime}\right\}$ is m-independent. $h_{a^{\prime} a} \llbracket b$, because $h_{a a^{\prime}} \in d c l\left(a a^{\prime}\right)$

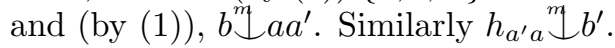

(3) We know that $h_{a^{\prime} a} \in \operatorname{acl}\left(b b^{\prime}\right)$, so by (1) we get that $h_{a^{\prime} a}{ }^{m} a, h_{a^{\prime} a}{ }^{m} a^{\prime}$ and $a^{m} \pm a^{\prime}$ by the choice of $a^{\prime}$.

Next, $h_{a^{\prime} a} \in \operatorname{dcl}\left(a a^{\prime}\right)$. We must show that $a \in \operatorname{acl}\left(h_{a^{\prime} a}, a^{\prime}\right)$ and $a^{\prime} \in \operatorname{acl}\left(h_{a^{\prime} a}, a\right)$. We have $a \in \operatorname{dcl}(b c), b \in \operatorname{acl}\left(b^{\prime}, h_{a^{\prime} a}\right)$ and $c \in d c l\left(a^{\prime} b^{\prime}\right)$, so we get $a \in \operatorname{acl}\left(a^{\prime}, b^{\prime}, h_{a^{\prime} a}\right)$.

$b^{\prime} \mathcal{I}^{m} a a^{\prime}$ gives $b^{\prime m}{ }^{m} a a^{\prime} h_{a^{\prime} a}\left(\right.$ as $\left.h_{a^{\prime} a} \in \operatorname{dcl}\left(a a^{\prime}\right)\right)$, so we get $a \in \operatorname{acl}\left(a^{\prime}, h_{a^{\prime} a}\right)$. Similarly we get $a^{\prime} \in \operatorname{acl}\left(a, h_{a^{\prime} a}\right)$.

The choice of the piece $h_{a a^{\prime}}$ of $g_{a a^{\prime}}$ such that Claim 3.4(2),(3) holds is the only place in the proof, where we use the m-normality of $U$.

Now we outline the further proof. We will find a clopen neighborhood $X_{5}$ of $a$ in $X$ such that the functions $h_{x x^{\prime}}=\left.g x x^{\prime}\right|_{Y_{0}}, x, x^{\prime} \in X_{5}$, form a definable group $H$ (the group operation is composition). In order to prove that the set $\left\{h_{x x^{\prime}}\right.$ : $\left.x, x^{\prime} \in X_{5}\right\}$ is closed under composition, we present each such $h_{x x^{\prime}}$ as a composition $\left(h_{x_{0} x}\right)^{-1} \circ h_{x_{0} x^{\prime}}$ for some $x_{0} \in X_{3}$, where $X_{3}$ is a clopen neighborhood of $a^{\prime}$ in $X . X_{3}$ and $X_{5}$ are chosen carefully by gradual restriction to smaller and smaller neighborhoods of $a$ and $a^{\prime}$ in $X$. The main point that we achieve in this way is that for $x^{\prime} \in X_{3}$ and $x \in X_{5}, h_{x^{\prime} x} \in o\left(h_{a^{\prime} a}\right)$, so such $h_{x^{\prime} x}$ may be treated uniformly (while there are infinitely many orbits on the set $\left\{h_{x^{\prime} x}: x, x^{\prime} \in X_{5}\right\}$ ).

Let $S=o\left(a^{\prime} a\right) \subseteq X \times X$. Since $\left.a^{m}\right\rfloor a^{\prime}$, we have that $S$ is a clopen subset of $X \times X$. For $\left(x^{\prime}, x\right) \in S$ let $h_{x^{\prime} x}$ be the conjugate of $h_{a^{\prime} a}$. Take a clopen neighbourhood $S_{0} \subseteq S \cap\left(X_{0} \times X_{0}\right)$ of $\left(a^{\prime}, a\right)$ such that for $\left(x^{\prime}, x\right) \in S_{0}, h_{x^{\prime} x}\left[Y_{0}\right]=h_{a^{\prime} a}\left[Y_{0}\right]$. Choose $E^{\prime} \in F E$ with $\left(\left[a^{\prime}\right]_{E^{\prime}} \cap X\right) \times\left([a]_{E^{\prime}} \cap X\right) \subseteq S_{0}$ and let $a^{+}=\left\langle a^{\prime} / E^{\prime}, a / E^{\prime}\right\rangle \in$ $U^{e q} \cap \operatorname{acl}(\emptyset)$. Let $X_{1}=\left[a^{\prime}\right]_{E^{\prime}} \cap X, X_{2}=[a]_{E^{\prime}} \cap X . X_{1}, X_{2} \subseteq X_{0}$. Clearly, $o\left(a^{\prime} a / a^{+}\right)=X_{1} \times X_{2}$.

Let $R=o\left(a^{\prime} a / a^{+} h_{a^{\prime} a}\right)$. We have that $\left\{a, a^{\prime}, h_{a^{\prime} a}\right\}$ is an $a c l$-triangle. So $R$ is a closed subset of $X_{1} \times X_{2}$, with all horizontal and vertical sections finite, and the projections to both coordinates open in $X$.

Let $X_{3}=o\left(a^{\prime} / a^{+} h_{a^{\prime} a}\right) \subseteq X_{1}$ and $X_{4}=o\left(a / a^{+} h_{a^{\prime} a}\right) \subseteq X_{2}$. So $X_{3}, X_{4}$ are the projections of $R$ to the respective coordinates, hence they are open in $X$.

Let $X_{3}^{+}, X_{4}^{+} \in \operatorname{acl}(\emptyset)$ be the names of $X_{3}, X_{4}$ and $a^{*}=\left\langle X_{3}^{+}, X_{4}^{+}\right\rangle$. Let $R^{\prime}=$ $o\left(a^{\prime} a / a^{+} a^{*}\right)$. Since $a^{*} \in d c l\left(a^{+} h_{a^{\prime} a}\right)$, we have that $R \subseteq R^{\prime} \subseteq X_{3} \times X_{4}$ and $R^{\prime}$ is clopen in $X_{3} \times X_{4}$. For $\left(x^{\prime}, x\right) \in R^{\prime}$ let $R_{x^{\prime} x}=o\left(x^{\prime} x / a^{+} a^{*} h_{x^{\prime} x}\right)$. Since $a^{+}, h_{x^{\prime} x} \in d c l\left(x^{\prime}, x\right)$, we have that $o\left(x x^{\prime} a^{+} a^{*} h_{x^{\prime} x}\right)=o\left(a a^{\prime} a^{+} a^{*} h_{a^{\prime} a}\right)$, hence

(**) $R_{x^{\prime} x}$ is a closed subset of $X_{3} \times X_{4}$, projecting onto $X_{3}$ and $X_{4}$, with all horizontal and vertical sections finite. Also, $R_{x^{\prime} x} \subseteq R^{\prime}$ and for $\left(t^{\prime}, t\right) \in R_{x^{\prime} x}, h_{t^{\prime} t}=$ $h_{x^{\prime} x}$.

Now we define a binary relation $\sim_{0}$ on $X_{4}$ :

$$
x_{1} \sim_{0} x_{2} \text { iff there is some } x^{\prime} \in X_{3} \text { with }\left(x^{\prime}, x_{1}\right),\left(x^{\prime}, x_{2}\right) \in R^{\prime} .
$$

Clearly, $\sim_{0}$ is symmetric and reflexive. Since $R^{\prime}$ is an open orbit, for some $E^{\prime \prime} \in F E$, for every $x \in X_{4}$, the set $\left\{x_{1} \in X_{4}: x \sim_{0} x_{1}\right\}$ is a union of $E^{\prime \prime}$-classes.

Let $\sim$ be the transitive closure of $\sim_{0}$ on $X_{4}$. We see that $\sim$ is an $a^{+} a^{*}$-definable equivalence relation on $X_{4}$, which is coarser than $E^{\prime \prime}$, hence has open classes. Also, 
there is some $n<\omega$ such that for every $x_{1}, x_{n} \in X_{4}$ we have

$$
x_{1} \sim x_{n} \Longleftrightarrow \exists x_{2}, \ldots, x_{n-1} \in X_{4} \bigwedge_{1 \leq i<n} x_{i} \sim_{0} x_{i+1} .
$$

Let $X_{5}$ be the $\sim$-class of $a$. For $x, x^{\prime} \in X_{5}$ let $h_{x x^{\prime}}=\left.g_{x x^{\prime}}\right|_{Y_{0}}$.

Lemma 3.5. $h_{x x^{\prime}}$ is a bijection mapping $Y_{0}$ onto $Y_{0}$.

Proof. We know that $x \sim x^{\prime}$. First we prove the lemma assuming additionally that $x \sim_{0} x^{\prime}$. This means that for some $x_{0} \in X_{3},\left(x_{0}, x\right),\left(x_{0}, x^{\prime}\right) \in R^{\prime}$. In particular,

$$
\left(x_{0}, x\right),\left(x_{0}, x^{\prime}\right) \in o\left(a^{\prime} a / a^{+}\right) \subseteq S_{0} .
$$

So by the choice of $S_{0}$ we have $h_{x_{0} x}\left[Y_{0}\right]=h_{x_{0} x^{\prime}}\left[Y_{0}\right]=$ some open $Y^{\prime} \subset Y$. We have

$$
g_{x x^{\prime}}=f_{x}^{-1} f_{x^{\prime}}=f_{x}^{-1} f_{x_{0}} f_{x_{0}}^{-1} f_{x^{\prime}}=g_{x_{0} x}^{-1} \circ g_{x_{0} x^{\prime}} .
$$

For any $\left(x_{1}, x_{2}\right) \in S_{0}, h_{x_{1} x_{2}}=\left.g_{x_{1} x_{2}}\right|_{Y_{0}}$, so

$$
h_{x x^{\prime}}\left[Y_{0}\right]=g_{x x^{\prime}}\left[Y_{0}\right]=g_{x_{0} x}^{-1}\left[g_{x_{0} x^{\prime}}\left[Y_{0}\right]\right]=g_{x_{0} x}^{-1}\left[h_{x_{0} x^{\prime}}\left[Y_{0}\right]\right]=g_{x_{0} x}^{-1}\left[Y^{\prime}\right]=h_{x_{0} x}^{-1}\left[Y^{\prime}\right]=Y_{0} .
$$

Now we approach the general case. $x \sim x^{\prime}$ means that there are $x_{1}, x_{2}, \ldots, x_{n} \in$ $X_{5}$ with $x=x_{1}, x^{\prime}=x_{n}$ and $x_{1} \sim_{0} x_{2} \sim_{0} \cdots \sim_{0} x_{n}$. We know that for every $i$ with $1 \leq i<n, h_{x_{i} x_{i+1}}$ is a bijection mapping $Y_{0}$ onto $Y_{0}$. So $h_{x_{1} x_{n}}=$ $h_{x_{1} x_{2}} h_{x_{2} x_{3}} \cdots h_{x_{n-1} x_{n}}$ also maps $Y_{0}$ onto $Y_{0}$.

Let $H=\left\{h_{x x^{\prime}}: x, x^{\prime} \in X_{5}\right\}$. We see that $H$ is a definable set of bijections: $Y_{0} \rightarrow$ $Y_{0}$.

Lemma 3.6. (1) For every $x, x^{\prime}, y^{\prime} \in X_{5}$ there is some $y \in X_{5}$ with $h_{x x^{\prime}}=h_{y y^{\prime}}$.

(2) $H$ is closed under composition of functions and under compositional inverse.

Proof. (1) $x^{\prime} \sim x$, so there are $x_{2}, \ldots, x_{n-1} \in X_{5}$ with $x^{\prime} \sim_{0} x_{2} \sim_{0} x_{3} \sim_{0} \cdots \sim_{0}$ $x_{n-1} \sim_{0} x$. Choose $t_{1}, \ldots, t_{n-1} \in X_{3}$ such that

$$
\left(t_{1}, x^{\prime}\right),\left(t_{1}, x_{2}\right),\left(t_{2}, x_{2}\right),\left(t_{2}, x_{3}\right), \ldots,\left(t_{n-1}, x_{n-1}\right),\left(t_{n-1}, x\right) \in R^{\prime} .
$$

By $(* *)$, choose recursively $y_{2}, \ldots, y_{n-1}, y \in X_{5}$ and $v_{1}, \ldots, v_{n-1} \in X_{3}$ with

$$
\begin{gathered}
\left(v_{1}, y^{\prime}\right) \in R_{t_{1} x^{\prime}},\left(v_{1}, y_{2}\right) \in R_{t_{1} x_{2}},\left(v_{2}, y_{2}\right) \in R_{t_{2} x_{2}},\left(v_{2}, y_{3}\right) \in R_{t_{2} x_{3}}, \ldots, \\
\left(v_{n-1}, y_{n-1}\right) \in R_{t_{n-1} x_{n-1}},\left(v_{n-1}, y\right) \in R_{t_{n-1} x} .
\end{gathered}
$$

By $(* *)$ we have

$$
\begin{gathered}
h_{t_{1} x^{\prime}}=h_{v_{1} y^{\prime}}, h_{t_{1} x_{2}}=h_{v_{1} y_{2}}, h_{t_{2} x_{2}}=h_{v_{2} y_{2}}, h_{t_{2} x_{3}}=h_{v_{2} y_{3}}, \ldots, \\
h_{t_{n-1} x_{n-1}}=h_{v_{n-1} y_{n-1}}, h_{t_{n-1} x}=h_{v_{n-1} y} .
\end{gathered}
$$

Now

$$
h_{x x^{\prime}}=h_{t_{n-1} x}^{-1} h_{t_{n-1} x_{n-1}} \ldots h_{t_{2} x_{3}}^{-1} h_{t_{2} x_{2}} h_{t_{1} x_{2}}^{-1} h_{t_{1} x^{\prime}}
$$

Also

$$
h_{y y^{\prime}}=h_{v_{n-1} y}^{-1} h_{v_{n-1} y_{n-1}} \ldots h_{v_{2} y_{3}}^{-1} h_{v_{2} y_{2}} h_{v_{1} y_{2}}^{-1} h_{v_{1} y^{\prime}}
$$

We see that $h_{x x^{\prime}}=h_{y y^{\prime}}$, as needed.

(2) Let $x, x^{\prime}, y, y^{\prime} \in X_{5}$. By (1), there is $x^{\prime \prime} \in X_{5}$ with $h_{x x^{\prime}}=h_{x^{\prime \prime} y}$. So $h_{x x^{\prime}} h_{y y^{\prime}}=h_{x^{\prime \prime} y} h_{y y^{\prime}}=h_{x^{\prime \prime} y^{\prime}} \in H$. Also $\left(h_{x x^{\prime}}\right)^{-1}=h_{x^{\prime} x} \in H$.

$H$ is a definable set and it is easy to see that composition and taking compositional inverse are definable on $H$ (one needs to check yet that their graphs are closed). So $H$ is a definable group. 
Lemma 3.6 shows that $H=\left\{h_{x a}: x \in X_{5}\right\}$. We prove that every element $h_{x a}$ of $H$ is interdefinable with $x$ over $a$, that is the $a$-definable function $x \mapsto h_{x a}$ is a bijection between $X_{5}$ and $H$.

Suppose not, which means that for some $x \neq x^{\prime} \in X_{5}$ we have $h_{x a}=h_{x^{\prime} a}$. Then necessarily $h_{x^{\prime} x}=h_{x^{\prime} a}\left(h_{x a}\right)^{-1}=i d_{Y_{0}}$. As in the beginning of the proof of 3.3, $\left.f_{x}\right|_{Y_{0}}$ and $\left.f_{x^{\prime}}\right|_{Y_{0}}$ are interdefinable with $x, x^{\prime}$ respectively. $h_{x^{\prime} x}=i d_{Y_{0}}$ means that $\left.f_{x}\right|_{Y_{0}}=\left.f_{x^{\prime}}\right|_{Y_{0}}$, which shows that $x, x^{\prime}$ are interdefinable. However $\operatorname{acl}(x) \cap X=\{x\}$, a contradiction.

So the $a$-definable bijection between $X_{5}$ and $H$ induces on $X_{5}$ a group structure definable over $a$ and some parameters from $\operatorname{acl}(\emptyset)$, that we have named so far in the proof. This finishes the proof of Theorem 3.3.

\section{Corollaries}

In this section we assume $U$ is a small profinite structure and $\mathcal{O}$ is a 0 -definable orbit in $U$, which has $\mathcal{M}$-rank 1 , is non-trivial and locally modular. In Section 3 we proved that there is an open definable set $\mathcal{O}^{\prime} \subseteq \mathcal{O}$, which is a group definable over some number of parameters. In this section we prove that in $U^{e q}$ there is a group $G$ of $\mathcal{M}$-rank 1, which is m-nonorthogonal to $\mathcal{O}$ and definable over $\operatorname{acl}(\emptyset)$. Also, we prove that for every $a \in \mathcal{O}$ there is a group structure on some open neighbourhood of $a$ in $\mathcal{O}$, which is definable just over $\{a\} \cup \operatorname{acl}(\emptyset)$, and moreover $a$ is the neutral element. We conclude the paper with an analysis of m-dependence in a locally modular definable group of $\mathcal{M}$-rank 1 . We prove that the division ring underlying the geometry on $\mathcal{O}$ is a locally finite field. We prove also that the acl-geometry on $\mathcal{O}$ cannot be modular.

We begin with a lemma describing m-nonorthogonality of locally modular orbits of $\mathcal{M}$-rank 1.

Lemma 4.1. Assume $U$ is m-stable or m-normal. Assume $X, Y$ are $\mathcal{M}$-rank 1 locally modular orbits over $\emptyset$ in $U$ and $X, Y$ are m-nonorthogonal. Then there are $x, x^{\prime} \in X$ and $y, y^{\prime} \in Y$ such that $x^{m^{\prime}} x^{\prime}, y^{m} \downarrow y^{\prime}$ and the set $\left\{x, x^{\prime}, y, y^{\prime}\right\}$ is m-dependent.

Proof. Considering the profinite structure induced on $X \cup Y$ we may assume that $U$ is m-normal (as in the beginning of Section 3). Choose a finite set $A \subseteq U$ and $x \in X, y \in Y$ generic over $A$ with $x^{m} \Psi y(A)$.

By m-normality, there is some $z \in \operatorname{acl}(x y) \cap \operatorname{acl}(A)$ with $x y^{n} \perp A(z)$. It follows that $\mathcal{M}(z)=1$ and $x^{m} \mathbb{Y} y(z)$. Choose $x^{\prime} y^{\prime} \in o(x y / z)$ with $x^{\prime} y^{\prime m} \Psi^{\prime} x y(z)$. Since $x^{m} \Psi_{z}$

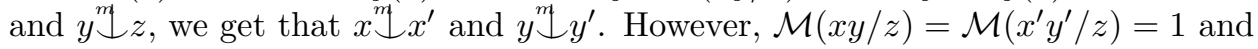
$z \in \operatorname{acl}(x y)$. So $\mathcal{M}\left(x y x^{\prime} y^{\prime}\right)=\mathcal{M}\left(x y x^{\prime} y^{\prime} z\right)=\mathcal{M}(x y / z)+\mathcal{M}\left(x^{\prime} y^{\prime} / z\right)+\mathcal{M}(z)=3$. So the set $\left\{x, y, x^{\prime}, y^{\prime}\right\}$ is m-dependent.

Before proving the next corollary we must analyze the acl-geometry on $\mathcal{O}$. Let $D$ be a countable dense subset of $\mathcal{O}$. The geometry on $\mathcal{O}$ is non-trivial, so for every $a \in \mathcal{O} \backslash \operatorname{acl}(D)$, the topological closure of $\operatorname{acl}_{D}(a)$ is clopen in $\mathcal{O}$. So on $\mathcal{O}$ we may define an equivalence relation $E$ by

$a E a^{\prime}$ iff for some countable dense $D \subseteq \mathcal{O}$ with $a, a^{\prime} \notin a c l(D)$, we have $\operatorname{cl}\left(\operatorname{acl}_{D}(a)\right)=\operatorname{cl}\left(\operatorname{acl}_{D}\left(a^{\prime}\right)\right)$.

Clearly, $E$ is a 0 -definable equivalence relation on $\mathcal{O}$, with finitely many classes. Let $\mathcal{O}_{1}, \ldots, \mathcal{O}_{n}$ be the $E$-classes. So $\mathcal{O}_{1}, \ldots, \mathcal{O}_{n}$ are orbits over elements of the form $a / E, a \in \mathcal{O}$. Also, $\mathcal{O}_{1}, \ldots, \mathcal{O}_{n}$ are $\mathrm{m}$-orthogonal, however each $\mathcal{O}_{i}$ is unidimensional 
in the following sense: if $X, Y$ are infinite orbits contained in $\mathcal{O}_{i}$, then $X, Y$ are m-nonorthogonal. For more details on this notion see [Ne3].

Corollary 4.2. Assume $U$ is a small profinite structure and $\mathcal{O}$ is a non-trivial $\mathcal{M}$-rank 1 locally modular orbit in $U$, over $\emptyset$. Then for every $a \in \mathcal{O}$ there is some clopen neighbourhood $\mathcal{O}^{\prime}$ of a in $\mathcal{O}$ and there is a group $H$ in $U^{e q}$ of $\mathcal{M}$-rank 1, definable over acl $(\emptyset)$, with a regular and transitive action on $\mathcal{O}^{\prime}$, definable over acl $(\emptyset)$. In particular, this action induces on $\mathcal{O}^{\prime}$ a group structure, definable over $\{a\} \cup \operatorname{acl}(\emptyset)$.

Proof. Wlog $\mathcal{O}$ is unidimensional and $U$ is m-normal, of finite $\mathcal{M}$-rank. By Lemma 3.1 , there is $n<\omega$ such that for every $x \in \mathcal{O},|\operatorname{acl}(x) \cap \mathcal{O}|=n$. After naming a sufficiently large finite subset of $\operatorname{acl}(\emptyset), \mathcal{O}$ splits into finitely many new orbits $\mathcal{O}_{1}, \ldots, \mathcal{O}_{k}$ (for some $k>n$ ), which are m-nonorthogonal. As $k>n$, some $\mathcal{O}_{i}$ and $\mathcal{O}_{j}$ are almost m-orthogonal.

We claim that there is an algebraic triangle $\{a, b, c\}$ with $\mathcal{M}(a)=\mathcal{M}(b)=$ $\mathcal{M}(c)=1$ and $a \in \mathcal{O}$.

Indeed, by Lemma 4.1 there are m-independent $a, b \in \mathcal{O}_{i}$ and m-independent $c, d \in \mathcal{O}_{j}$ such that the set $\{a, b, c, d\}$ is m-dependent. So $a b^{m}\{c d$. By m-normality, there is some $e \in \operatorname{acl}(a b) \cap a c l(c d)$ with $a b^{m}\left\lfloor c d(e)\right.$. Using the fact that $\mathcal{O}_{i}$ and $\mathcal{O}_{j}$ are almost m-orthogonal it is easy to show that $\mathcal{M}(e)=1$ and then either $\{a, b, e\}$ or $\{c, d, e\}$ is an acl-triangle.

Given an algebraic triangle $\{a, b, c\}$ with $a \in \mathcal{O}$, in Section 3 we constructed a group $H$ definable over acl $(\emptyset)$, m-nonorthogonal to $\mathcal{O}$, with $\mathcal{M}(H)=1$. Also, $X_{5}$ there is a clopen subset of $\mathcal{O}$ and the mapping $\left(h, x^{\prime}\right) \mapsto x$, where $h \in H, x, x^{\prime} \in X_{5}$ and $h_{x x^{\prime}}=h$, is a definable regular transitive action of $H$ on $X_{5}$. This proves the corollary.

For further corollaries we need some facts about small m-normal profinite groups.

Theorem 4.3 ([Ne4] $)$. Assume $U$ is m-normal and $G$ is a group 0-definable in $U^{e q}$. Then $G$ is abelian-by-finite. Moreover, for every finite set $A$ and every $a \in G$, the orbit $o(a / A)$ is a finite union of cosets of a subgroup $H$ of $G$ definable over $\operatorname{acl}(\emptyset)$.

Corollary 4.4. Assume $U$ is m-normal and $G_{1}, G_{2}$ are 0 -definable groups in $U^{e q}$, of $\mathcal{M}$-rank 1. If $G_{1}, G_{2}$ are m-nonorthogonal, then there is a function $f$ definable over acl $(\emptyset)$, which is an isomorphism between open subgroups of $G_{1}$ and $G_{2}$.

Proof. Choose $A \subseteq U^{e q}$ and $A$-generic $a \in G_{1}$ and $b \in G_{2}$ with $a^{m}\lfloor b(A)$. Since $\mathcal{M}\left(G_{1}\right)=\mathcal{M}\left(G_{2}\right)=1$, this means that $a, b$ are interalgebraic over $A$. Extending $A$ by a finite subset of $a c l(\emptyset)$ we may assume that $a, b$ are interdefinable over $A$. By Theorem 4.3 there is a subgroup $H$ of $G_{1} \times G_{2}$, definable over $\operatorname{acl}(\emptyset)$, such that $o(a b / A)$ is a finite union of cosets of $H$. It follows that $H$ is the graph of an isomorphism $f$ between open subgroups of $G_{1}$ and $G_{2}$.

Corollary 4.5. Assume $U$ is m-stable or $m$-normal and $\mathcal{O}_{1}, \mathcal{O}_{2}$ are locally modular $\mathcal{M}$-rank 1 orbits over $\emptyset$ in $U$. Assume $\mathcal{O}_{1}, \mathcal{O}_{2}$ are m-nonorthogonal.

(1) If $\mathcal{O}_{1}, \mathcal{O}_{2}$ are trivial, then for some $a_{1} \in \mathcal{O}_{1}$ and $a_{2} \in \mathcal{O}_{2}$, there is a function $f$ definable over acl $(\emptyset)$ with $f\left(a_{1}\right)=a_{2}$, which is a bijection between some open neihbourhoods of $a_{i}$ in $\mathcal{O}_{i}$. 
(2) If $\mathcal{O}_{1}, \mathcal{O}_{2}$ are non-trivial and unidimensional, then for every $a_{1} \in \mathcal{O}_{1}$ and $a_{2} \in \mathcal{O}_{2}$, a function $f$ as in (1) exists, only $f$ is definable over $\left\{a_{1}, a_{2}\right\} \cup \operatorname{acl}(\emptyset)$.

Proof. We may assume $U$ is m-normal. (1) is easy. (2) Choose open $G_{a_{1}} \subseteq \mathcal{O}_{1}$ and $G_{a_{2}} \subseteq \mathcal{O}_{2}$, which are groups definable over $\left\{a_{i}\right\} \cup \operatorname{acl}(\emptyset)$, with neutral elements $a_{1}$ and $a_{2}$. The required function exists by Corollary 4.4 .

Next we analyze m-dependence in an m-normal group of $\mathcal{M}$-rank 1 . So assume $G$ is a 0 -definable $\mathcal{M}$-rank 1 group in $U$, which is locally modular (that is, any generic orbit of $G$ is locally modular). So wlog we may assume that $U$ is $\mathrm{m}$ normal and of finite $\mathcal{M}$-rank. Since $G$ is abelian-by-finite, we may assume that $G$ is abelian. Similarly as in $[\mathrm{H}]$ we define a division ring $\mathcal{F}_{G}$ of germs of definable local endomorphisms of $G$.

We say that $f$ is a definable local endomorphism of $G$, if $f$ is a group homomorphism mapping some open subgroup of $G$ into $G$, and $f$ is definable. We say that two definable local endomorphisms $f, g$ are equivalent $(f \sim g)$, if they agree on an open subgroup of $G$. So the $\sim$-classes are germs at 0 of local endomorphisms. Moreover, by Theorem 4.3, every definable local endomorphism is equivalent to another local endomorphism definable over $\operatorname{acl}(\emptyset)$, so we may restrict ourselves only to such local endomorphisms. Let $\mathcal{F}_{G}$ be the ring of the $\sim$-classes of local endomorphisms definable over $\operatorname{acl}(\emptyset)$ (that is, the ring of germs at 0 of such endomorphisms). It is easy to see that $\mathcal{F}_{G}$ is a division ring.

In $[\mathrm{H}]$, forking was described locally on the connected component of a locally modular regular group as linear dependence over the ring of definable pseudoendomorphisms. Here $G^{0}=\{0\}$, so we must localize $G$ in a different way. We have that for every finite $A, \operatorname{acl}(A) \cap G$ is finite (see [Ne6, Proposition 2.4]).

Let $D$ be a countable dense acl-closed subgroup of $G$. Then $\mathcal{F}_{G}$ acts on $G / D$ :

$$
\begin{aligned}
& \text { for } a+D \in G / D \text { and } f^{\prime}=f / \sim \in \mathcal{F}_{G} \text {, let } f^{\prime}(a+D)=f\left(a^{\prime}\right)+D \text {, where } \\
& a^{\prime} \in(a+D) \cap \operatorname{dom}(f) .
\end{aligned}
$$

This definition does not depend on the choice of $a^{\prime}$ and $f$ in the class $f / \sim$. Indeed, suppose $a^{\prime \prime} \in(a+D) \cap \operatorname{dom}(f)$. Then $a^{\prime}-a^{\prime \prime} \in D$, hence $f\left(a^{\prime}-a^{\prime \prime}\right)=f\left(a^{\prime}\right)-f\left(a^{\prime \prime}\right) \in$ $\operatorname{acl}(D) \cap G=D$, so $f\left(a^{\prime}\right)+D=f\left(a^{\prime \prime}\right)+D$. Also, $G / D$ is a vector space over $\mathcal{F}_{G}$.

Theorem 4.6. acl $_{D}$-dependence on $G / D$ is the linear dependence over $\mathcal{F}_{G}$.

Proof. If $a_{1}+D, \ldots, a_{n}+D$ are linearly dependent over $\mathcal{F}_{G}$, then $a_{1}, \ldots, a_{n}$ are $\operatorname{acl}_{D}$-dependent.

For the other direction, assume $a_{1}+D, \ldots, a_{n}+D$ are $a c l_{D}$-dependent, that is (more formally) $a_{1}, \ldots, a_{n}$ are $a c l_{D}$-dependent. We will prove that $a_{1}+D, \ldots, a_{n}+$ $D$ are linearly dependent over $\mathcal{F}_{G}$. Choose $d_{n+1}, \ldots, d_{n+k} \in D$ such that $a_{1}, \ldots, a_{n}$, $d_{n+1}, \ldots, d_{n+k}$ are acl-dependent. We may assume that any proper subset of $\left\{a_{1}, \ldots, a_{n}, d_{n+1}, \ldots, d_{n+k}\right\}$ is $a c l$-independent.

Let $V=o\left(a_{1}, \ldots, a_{n}, d_{n+1}, \ldots, d_{n+k}\right) \subseteq G^{n+k}$. By Theorem 4.3, possibly naming some parameters from $\operatorname{acl}(\emptyset)$, we may assume that $V$ is a coset of some 0definable subgroup $H$ of $G^{n+k}$. For $1 \leq i \leq n+k$, let $\pi_{\neq i}: G^{n+k} \rightarrow G^{n+k-1}$ be defined by $\pi_{\neq i}\left(x_{1}, \ldots, x_{n+k}\right)=\left(x_{1}, \ldots, x_{i-1}, x_{i+1}, \ldots, x_{n+k}\right)$, and let $H_{\neq i}=\pi_{\neq i}[H]$, this is an open subgroup of $G^{n+k-1}$. Moreover we may assume that for every $\bar{x} \in H_{\neq i},\left|\pi_{\neq i}^{-1}(\bar{x}) \cap H\right|=1$.

For $1<i \leq n+k$ let $H_{i}=\left\{\left(x_{1}, 0, \ldots, 0, x_{i}, 0, \ldots, 0\right) \in H: x_{1}, x_{i} \in G\right\}$. As in [H] we see that $H_{2}+\cdots+H_{n+k}$ is an open subgroup of $H$ and each $H_{i}$ 
corresponds to the graph of a local endomorphism $f_{i}$ with $f_{i}\left(x_{i}\right)=x_{1}$ (where $\left.\left(x_{1}, 0, \ldots, 0, x_{i}, 0, \ldots, 0\right) \in H_{i}\right)$.

Choose $d_{1}^{\prime}, \ldots, d_{n+k}^{\prime} \in D$ with

$$
\left(a_{1}-d_{1}^{\prime}, \ldots, a_{n}-d_{n}^{\prime}, d_{n+1}-d_{n+1}^{\prime}, \ldots, d_{n+k}-d_{n+k}^{\prime}\right) \in H_{2}+\cdots+H_{n+k} .
$$

It follows that $a_{1}+D=\sum_{i=2}^{n} f_{i}\left(a_{i}+D\right)$, so we are done.

Remark 4.7. $\mathcal{F}_{G}$ is a locally finite field.

Proof. The proof is the same as in [Ne1, Corollary 2.5(1)], in the case of a meager group. The main point is that for every finite set $\mathcal{F}$ of local endomorphisms of $G$ definable over $\operatorname{acl}(\emptyset)$, there is an open subgroup $G^{\prime}$ of $G$ contained in the domains of all the functions from $\mathcal{F}$, and closed under them. Let $\hat{\mathcal{F}}$ be the ring of functions generated by $\mathcal{F}$, restricted to $G^{\prime}$. Since for any generic $a \in G^{\prime}$ we have $\{f(a): f \in$ $\hat{\mathcal{F}}\} \subseteq \operatorname{acl}(a) \cap G^{\prime}$, and the latter set is finite, we conclude that $\hat{\mathcal{F}}$ is finite.

Corollary 4.8. Assume $U$ is a small profinite structure and $\mathcal{O}$ is a non-trivial locally modular $\mathcal{M}$-rank 1 orbit over $\emptyset$ in $U$. Then the division ring associated with the geometry on $\mathcal{O}$ is a locally finite field.

Proof. Let $\mathcal{O}_{1}, \ldots, \mathcal{O}_{n}$ be the partition of $\mathcal{O}$ into unidimensional pairwise m-orthogonal orbits. After suitable dense localization, the acl-geometry on $\mathcal{O}$ is the disjoint union of the geometries on $\mathcal{O}_{1}, \ldots, \mathcal{O}_{n}$, and the geometry on each $\mathcal{O}_{i}$ is projective over some division $\operatorname{ring} \mathcal{F}_{i}$. In fact, the rings $\mathcal{F}_{1}, \ldots, \mathcal{F}_{n}$ are isomorphic, so we may treat them as a single ring $\mathcal{F}$, underlying the geometry on $\mathcal{O}$.

We may assume that $\mathcal{O}$ is unidimensional and that there is an $\mathcal{M}$-rank 10 definable group $G$ m-nonorthogonal to $\mathcal{O}$. Then after suitable dense localization, the geometry on $\mathcal{O}$ is isomorphic to the acl-geometry on $G$, which is projective over $\mathcal{F}_{G}$. So $\mathcal{F}$ is isomorphic to $\mathcal{F}_{G}$. By Remark $4.7, \mathcal{F}$ is locally finite.

If $p$ is a regular stationary non-trivial locally modular type in some stable theory $T$, then the forking geometry on $p$ is either affine (when $p$ is not modular) or projective (when $p$ is modular) over some division ring $[\mathrm{H}]$.

In small profinite structures the picture is more complicated. When the field $\mathcal{F}$ (from Corollary 4.8) is infinite, then the acl-geometry on $\mathcal{O}$ can be neither affine nor projective over $\mathcal{F}$, because $\operatorname{acl}(A) \cap \mathcal{O}$ is finite for every finite $A \subseteq \mathcal{O}$.

Corollary 4.9. When the field $\mathcal{F}$ underlying the geometry on $\mathcal{O}$ is finite, then there is an open orbit $\mathcal{O}^{\prime \prime} \subseteq \mathcal{O}$ (over some parameters from acl $(\emptyset)$ ) such that the acl-geometry on $\mathcal{O}^{\prime \prime}$ is affine over $\mathcal{F}$.

Proof. Let $H$ and $\mathcal{O}^{\prime}$ be as in Corollary 4.2. Wlog $H$ and $\mathcal{O}^{\prime}$ are 0 -definable. Since $\mathcal{F}$ is finite, by the proof of Remark 4.7, some open 0-definable subgroup $H^{\prime}$ of $H$ is an $\mathcal{F}$-vector space. $H^{\prime}$ acts regularly and transitively on some open $\mathcal{O}^{\prime \prime} \subseteq \mathcal{O}^{\prime}$. Hence the acl-geometry on $\mathcal{O}^{\prime \prime}$ is affine over $\mathcal{F}$.

Unlike in stable model theory, we have the following corollary.

Corollary 4.10. There is no non-trivial $\mathcal{M}$-rank 1 orbit $\mathcal{O}$ in a small profinite structure $U$, such that the acl-geometry on $\mathcal{O}$ is modular.

To prove this corollary we need a stronger version of Lemma 3.1. Essentially it is [Ne2, Lemma 2.1]. We repeat the proof here.

Lemma 4.11. Assume $U$ is a small profinite $m$-stable structure, $A \subseteq U^{e q}$ is finite and $X$ is an orbit in $U^{\text {eq }}$ of $\mathcal{M}$-rank 1. Then acl $(A) \cap X$ is finite. 
Proof. Wlog (possibly after naming some parameters) we may assume that $X$ is an orbit over $\emptyset$. Since $\mathcal{M}(X)=1$, acl is a pregeometry on $X$.

Every type in a superstable theory has finite weight. Here similarly m-stability implies that there is a finite set $B \subseteq X \cap \operatorname{acl}(A)$ such that $B$ is an acl-basis of $X \cap \operatorname{acl}(A)$.

So we may assume that $A \subseteq X$. Suppose for a contradiction that the lemma is false. Choose $A \subseteq X$ with $\operatorname{acl}(A) \cap X$ infinite and such that $A$ has minimal size. This implies that $A$ is a basis of $\operatorname{acl}(A) \cap X$.

Case 1. Assume some $a \in \operatorname{acl}(A) \cap X$ is an accumulation point of $\operatorname{acl}(A) \cap X$. Replacing $A$ by a basis of $\operatorname{acl}(A) \cap X$ containing $a$, we may assume that $a \in A$. Let $A^{\prime}=A \backslash\{a\}$ and $X^{\prime}=o\left(a / A^{\prime}\right)$. Since $A$ is m-independent, $X^{\prime}$ is open in $X$. Also, $\operatorname{acl}(A) \cap X^{\prime}$ is infinite. So naming $A^{\prime}$ and replacing $X$ by $X^{\prime}$ we may assume that $A=\{a\}$. Since all the elements of $X$ are conjugate, it follows that every $a^{\prime} \in \operatorname{acl}(a) \cap X$ is an accumulation point of $\operatorname{acl}(a) \cap X$.

Let $Y$ be the topological closure of $\operatorname{acl}(a) \cap X$. We get that $Y$ is a perfect set, of power $2^{\aleph_{0}}$. Also, $Y$ is a union of countably many orbits over a (by smallness), so some of these orbits must be infinite, hence open in $X$ (as $\mathcal{M}(X)=1)$. But open orbits over $a$ are disjoint from $\operatorname{acl}(a) \cap X$, a contradiction.

Case 2. Assume that no $a \in \operatorname{acl}(A) \cap X$ is an accumulation point of $\operatorname{acl}(A) \cap X$. Still, by compactness, there is some $a \in X \backslash \operatorname{acl}(A)$ which is an accumulation point of $\operatorname{acl}(A) \cap X . o(a / A)$ is infinite, hence is open in $X$ and disjoint from $\operatorname{acl}(A)$. So $a$ can not be an accumulation point of $\operatorname{acl}(A) \cap X$.

When $A \subseteq X$, then in Lemma 4.11 we may waive the m-stability assumption. If $U$ is m-normal and $\mathrm{m}$-stable (hence of finite $\mathcal{M}$-rank), then Lemma 4.11 is true also when $\mathcal{M}(X)>1$ (the proof uses full coordinatization of $U$ ). In general it is open if Lemma 4.11 remains true when $\mathcal{M}(X)>1$.

Proof of 4.10. Suppose for a contradiction, that the acl-geometry is modular on $\mathcal{O}$. This implies also that for every dense $D \subseteq \mathcal{O}$, the $a c l_{D}$-geometry on $\mathcal{O}$ is modular, so $\mathcal{O}$ is locally modular, with an underlying field $\mathcal{F}$. By the discussion before Corollary $4.9, \mathcal{F}$ is finite.

We define an equivalence relation $E$ on $\mathcal{O}$ by: $E(x, y) \Longleftrightarrow x, y$ are interalgebraic. By Lemma 4.11, $E$ has finite classes. It follows that $E$ is 0-definable. Replacing $\mathcal{O}$ by $\mathcal{O} / E$ we may assume that for $x \in \mathcal{O}$, acl $(x) \cap \mathcal{O}=\{x\}$.

Partitioning $\mathcal{O}$ into pairwise $\mathrm{m}$-orthogonal unidimensional orbits, we may assume that $\mathcal{O}$ is unidimensional. This in turn yields that the acl-geometry on $\mathcal{O}$ is projective over $\mathcal{F}$. Choose $\mathcal{O}^{\prime \prime}$ from Corollary 4.9.

By Lemma 4.11, if $x, y \in \mathcal{O}^{\prime \prime}$ are sufficiently close to each other, then $\operatorname{acl}(x, y) \cap$ $\mathcal{O} \subseteq \mathcal{O}^{\prime \prime}$. Choose m-independent $a, b, c \in \mathcal{O}^{\prime \prime}$ sufficiently close to each other so that $a c l(a, b, c) \cap \mathcal{O} \subseteq \mathcal{O}^{\prime \prime}$.

So $\operatorname{acl}(a, b, c) \cap \mathcal{O}$ is a projective plane $\Pi$ over $\mathcal{F}$ in the acl-geometry on $\mathcal{O}$, and $\Pi \subseteq \mathcal{O}^{\prime \prime}$. On the other hand, the acl-geometry on $\mathcal{O}^{\prime \prime}$ is affine over $\mathcal{F}$, so on $\Pi$ there are disjoint (parallel) lines, a contradiction.

On the other hand, the acl-geometry is modular on the group $H^{\prime}$ from the proof of Corollary 4.9. So in small profinite structures locally modular groups seem the best approximation to modular types.

Now assume $T$ is a superstable theory with $<2^{\aleph_{0}}$ countable models and $U$ is a small profinite structure interpretable in $T$ (so $U$ is m-normal, with finite $\mathcal{M}$ rank). Assume $\mathcal{O}$ is an $\mathcal{M}$-rank 1 orbit definable in $U$. Then $\mathcal{O}$ is locally modular 
and non-trivial. Also, in $\mathrm{Ne} 3$ I proved that associated with $\mathcal{O}$ is a meager type $p$, and the field $\mathcal{F}$ underlying the forking geometry on $p$ corresponds also to the acl-geometry on $\mathcal{O}$. Then I proved Theorem 1.7 in this case. However, the results of this paper give some new information even here. In particular, the fact that an essentially 1 parameter from $\mathcal{O}$ is enough to define a group in it is new.

\section{REFERENCES}

[B] S.Buechler, Essential Stability Theory, Springer 1996. MR 98j:03050

[BH] E.Bouscaren, E.Hrushovski, On one-based theories, J.Symb.Logic 53(1994), 579-595. MR 95e:03103

[H] E.Hrushovski, Locally modular regular types, in: Classification Theory, Proceedings, Chicago 1985, ed. J.T.Baldwin, Springer 1987, 132-164. MR 90m:03064.

[Ne1] L.Newelski, Meager forking, Ann. Pure Appl.Logic 70(1994), 141-175. MR 96a:03047

[Ne2] L.Newelski, M-gap conjecture and m-normal theories, Israel J.Math. 106(1998), 285-311. MR 2000b:03122

[Ne3] L.Newelski, Geometry of *-finite types, J.Symb.Logic 64(1999), 1375-1395. CMP 2000:17

[Ne4] L.Newelski, m-normal theories, Fund.Math., accepted.

[Ne5] L.Newelski, Meager forking and m-independence, in: ICM'98 Proceedings, vol.II, 33-42. MR 2000a:03054

[Ne6] L.Newelski, Small profinite groups, J.Symb. Logic, accepted.

[Pe] Y.Peterzil, Constructing a group interval in o-minimal structures, J.Pure Appl.Algebra (1994), 85-100. MR 95h:03085

Mathematical Institute, University of Wroclaw, Pl. Grunwaldzki 2/4, 50-384 WroCLAW, POLAND

E-mail address: newelski@math.uni.wroc.pl

Mathematical Institute of the Polish Academy of Sciences, Kopernika 18, 51-617 Wroclaw, Poland 